Research Article

\title{
Experimental Study on Stress Wave Propagation Crossing the Jointed Specimen with Different JRCs
}

\author{
S.N. Hong $\mathbb{D}^{1,2}$ H.B. Li ${ }^{1},{ }^{1}$ and L.F. Rong ${ }^{3}$ \\ ${ }^{1}$ State Key Laboratory of Geomechanics and Geotechnical Engineering, Institute of Rock and Soil Mechanics, \\ Chinese Academy of Sciences, Wuhan 430072, China \\ ${ }^{2}$ University of Chinese Academy of Science, Beijing 100049, China \\ ${ }^{3}$ Key Laboratory of Structural Health Monitoring and Control, Shijiazhuang Tiedao University, Shijiazhuang 050043, China \\ Correspondence should be addressed to H.B. Li; hbli@whrsm.ac.cn
}

Received 7 July 2021; Accepted 30 August 2021; Published 3 November 2021

Academic Editor: Yujing Jiang

Copyright (C) 2021 S.N. Hong et al. This is an open access article distributed under the Creative Commons Attribution License, which permits unrestricted use, distribution, and reproduction in any medium, provided the original work is properly cited.

\begin{abstract}
Most of the rock masses in the outer crust of the Earth are discontinuous. They are divided by joints, faults, fractures, etc. And those discontinuities, generally referred to as joints, greatly affect the property of the rock masses. The paper experimentally investigates the stress wave propagation crossing the jointed specimens. The tests were conducted on the split Hopkinson pressure bar (SHPB). The test specimens consist of two parts cast by cement mortar. Both parts have an irregular surface, and they were designed to match each other completely. The surfaces where two parts meet make an artificial joint. The surfaces of the joints were scanned by a three-dimensional scanner to obtain its actual topography and then to calculate the roughness of the surface, i.e., the joint roughness coefficient $(J R C)$. A set of jointed specimens with $J R C$ ranging from 0 to 20 were made and used in dynamic compression experiments. During the tests, signals were captured by strain gauges stuck on the incident and transmitted bars of the SHPB apparatus. The incident, reflected, and transmitted waves across the jointed specimens were obtained from the test records. We found out that more stress wave would transmit through the jointed specimen with larger JRC. Besides, collected data were processed to get the dynamic stress-strain relation of jointed specimens and the stress-closure curves of the joints. The results show that the joint increases the deformation of the specimen, and the stiffness of the jointed specimen would increase slightly when the joint is rougher.
\end{abstract}

\section{Introduction}

Geological resources serve as the basis for modern life society. They are so crucial that many researchers work on relevant projects, such as mining, geological exploration, and geomechanics $[1,2]$. Among those projects, geomechanics is the foundation of them, which involves the study of the mechanics of soil and rock. And this paper focuses on the jointed rock under dynamic load.

Research shows that the rock joints significantly affect the mechanical behaviors of rock masses [3-5]. To quantify the joint effects on rock masses, we usually study the joint mechanical behavior first. Quite a few models and assumptions were proposed to characterize the joint mechanical behavior. One of the joint models is called the
Bandis-Barton model (B-B model) that was put forward by [6] based on quantities of static experiments. It is a hyperbolic function describing the stress-closure curves of joints in normal static loading, which is commonly accepted and used to describe the deformation of the joint. The normal stiffness $k_{n}$ and maximum normal joint closure $v_{\max }$ are considered as characteristic parameters to describe the stress-closure relations of the joint under normal compressive loading. However, the test results of [7] showed that the dynamic stiffness of rock joints differed from the static stiffness, and the former one was always higher than the latter. Furthermore, [8] modified the B-B model by taking the loading-rate into account. In another aspect, it is generally accepted that the joint is considered as an imperfectly bonded interface when a 
stress wave propagates upon the joint. A joint theory introduced by [9] was referred to as the displacement discontinuity method (DDM) as the displacement across the joint is not continuous, but the traction is continuous. With the method, reflection and transmission coefficients for stress waves incident upon a joint could be assessed in terms of interface compliance $[7,9]$. Later an equivalent viscoelastic medium model was proposed for determining the wave transmission through parallel joints [10]. Recently, [11] did a research on three creep stages of shear creep tests and proposed a method for predicting the accelerating creep stage considering the effect of the joint roughness.

The surfaces of the joints in nature are rough on some scale, and the contact condition of two rough joint surfaces affects most physical properties [12-14]. To explore the influence of the joint surface on its properties, it is of great value to quantify joint topography at first. There are kinds of ways that were proposed to quantify joint topography. And among them, the joint roughness coefficient $(J R C)$ is widely used for rock engineering applications. It was first recommended by [15] who gave a set of 10 typical roughness profiles with $J R C$ ranging from 0 to 20 . However, it is too subjective to estimate the JRC by comparing the joint surface to the 10 typical roughness profiles. Hence, Tse and Cruden [16] put forward an empirical equation to calculate $J R C$ correlating with $Z_{2}$ (the root mean square of the first derivative of the profile) and $S F$ (structure function). Then it was improved by [17] considering the self-affinity transformation law. The initial purpose of quantifying joint topography using $J R C$ is to illustrate the effect of joint roughness on peak shear strength, yet the joint roughness also affects the stress wave propagation. Reference [18] found that the joint closure behavior under dynamic load is highly nonlinear, which is complicatedly affected by rock joint roughness and initial closure. Besides, Li, Rong et al. [19] discovered that more stress wave energy dissipated in the jointed specimen with larger $J R C$ based on a series of laboratory tests.

The subject of this study is to experimentally investigate how $J R C$ affects the behavior of joint under dynamic compression and the stress wave propagation crossing the jointed specimen. The split Hopkinson pressure bar (SHPB) apparatus was adopted to conduct uniaxial compression experiments on specimens with rough joints. Jointed specimens were made by casting cement mortar into custom-made molds. Though the surfaces of the joint could be well controlled by specifying mold shape, they were scanned by a three-dimensional scanner to obtain its actual topography and then to calculate $J R C$. All jointed specimens and one intact specimen were put into dynamic compression tests. Based on the collecting data captured by strain gauge in tests, the incident, reflected, and transmitted waves were obtained and processed to get the stress and strain of the jointed specimens. Then the effect of JRC on the stress wave propagating across the jointed specimen and the dynamic mechanical behavior of the joint are analyzed and discussed in the end.

\section{Experiments}

2.1. Experimental Facilities. As shown in Figure 1, the uniaxial compression test was conducted on the SHPB system. It consists of loading system, bar system, and measurement system. The loading system is a gas gun, which stores high-pressure nitrogen that pushes the bullet to move forward. The bar system contains four steel bars with the same circle cross section ( $50 \mathrm{~mm}$ in diameter), namely bullet (400 $\mathrm{mm}$ in length), the incident bar (2500 $\mathrm{mm}$ in length), the transmitted bar (2500 $\mathrm{mm}$ in length), and the absorption bar $(800 \mathrm{~mm}$ in length). In addition, the density $\rho$ and Young's modulus $E$ of the steel bars are $7800 \mathrm{~kg} / \mathrm{m}^{3}$ and $210 \mathrm{GPa}$, respectively. The measurement system is made up of dynamic strain meter, oscilloscope, and two groups of strain gauges stuck on the incident and transmitted bar.

During the test, a specimen was coaxially sandwiched between the incident bar and the transmitted bar. When the bullet was shot out of the gas gun and impacted the incident bar, a compressive pulse was generated and propagated along the bar. As soon as the stress pulse reached the interface of incident bar and specimen, part of it was reflected in the incident bar, whereas the remaining part traveled through the specimen and transmitted into the transmission bar. Those stress pulses, named incident wave, reflected wave, and transmitted wave, respectively, were measured by strain gauges stuck on the incident and transmitted bars and then collected by dynamic strain meter and shown and stored by an oscilloscope.

2.2. Specimens. We adopted the random fractal method suggested by [20] to simulate natural joint profiles. The method uses the principle of Brownian motion. According to the method, we generated ten profile curves with different $J R C s$ by giving different Hurst exponent $H$. The profile curves are shown in Figure 2. Each profile curve was stretched linearly into a rough surface, which is rough in the horizontal direction and straight in the vertical direction and is called a two-dimensional rough surface. The rough surfaces were used to print 3D molds to cast specimens.

As for specimen preparation, first, cement, water, and sand were well mixed with a ratio of $1: 1: 0.4$ and then the mixture was cast into the molds. Cured for 28 days to reach the full strength, the cement specimens were then polished and got dry naturally. The specimens were cuboids with $3 \mathrm{~cm}$ in height and $3.5 \mathrm{~cm}$ in length and width. As shown in Figure 3, the specimens consisted of two parts cast. Each part has a rough bottom. The two rough bottoms of both parts match each other. The interface where two parts meet simulates an artificial rough joint. Besides, an intact specimen without joint was cast in the same material as a comparison to those jointed specimens.

The range of JRCs of specimens was designed to be between close to 0 and nearly 20 . But the roughness coefficients of printed specimens may have nuances from originally designed ones because of error brought by the molds and particle size of the material. Hence, the joint surfaces of specimens were scanned on the $3 \mathrm{D}$ scanner 


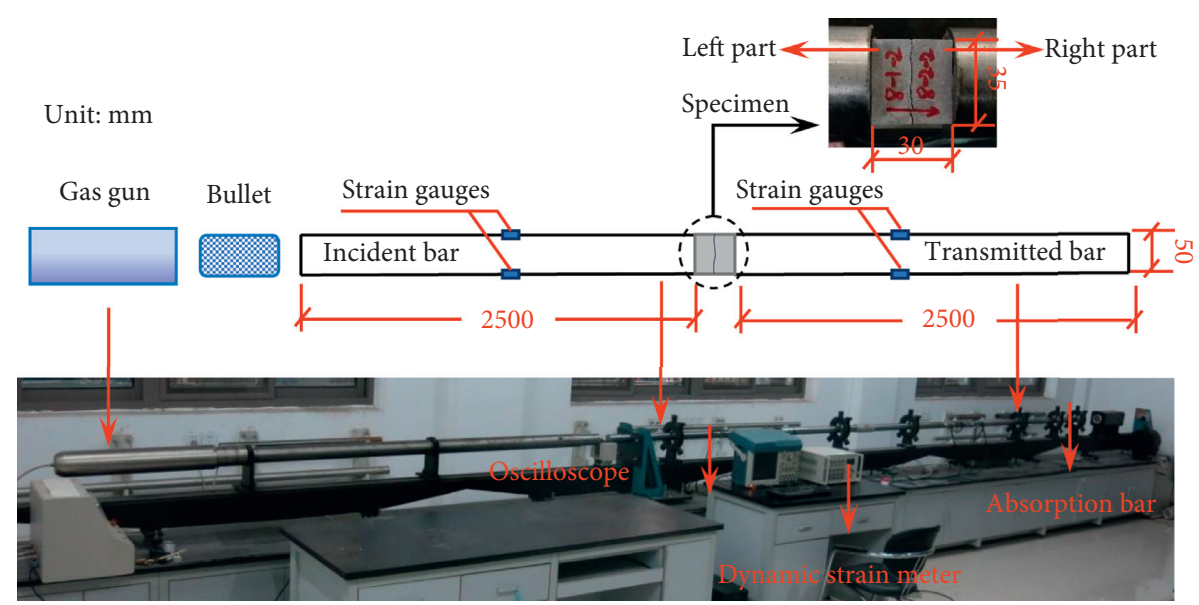

FIgURE 1: The split Hopkinson pressure bar (SHPB) system.

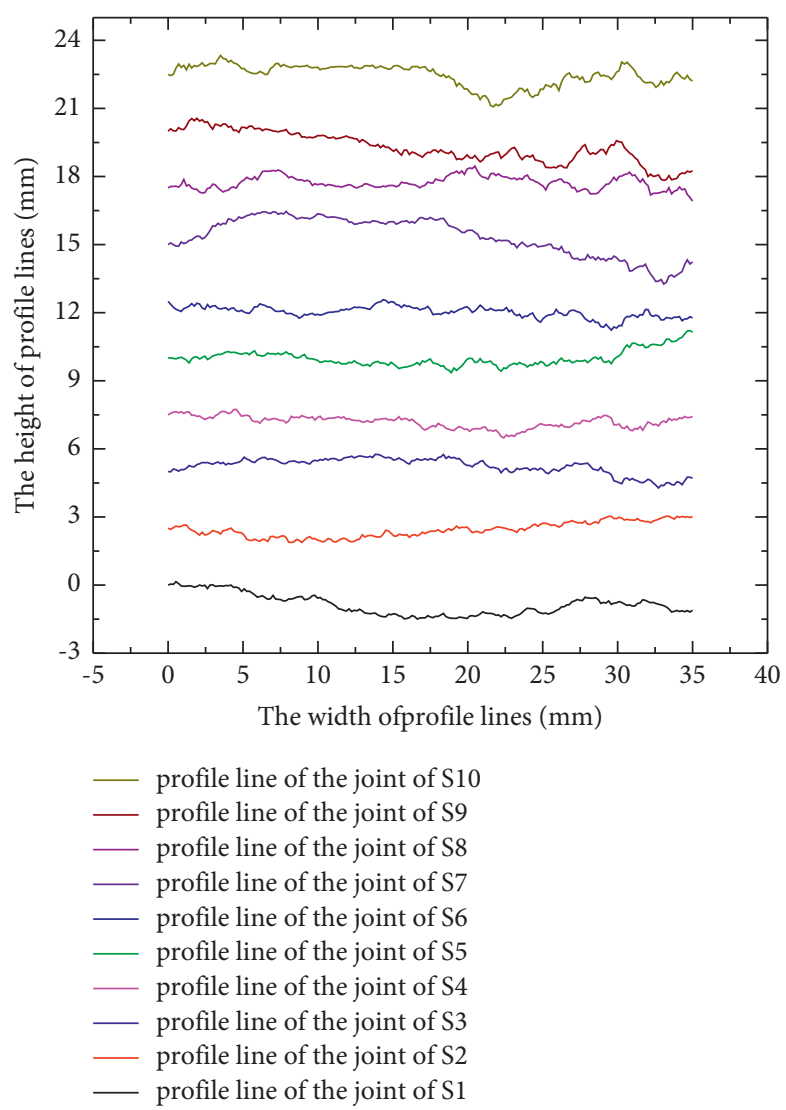

Figure 2: Profile lines of the surfaces of joints of the specimens.

(a type of Geomagic Capture) to obtain the actual 3D digital images of the joint and calculate the actual value of the JRC. Both designed values and actual values of $J R C$ are displayed in Table 1 and Figure 4.

It can be seen that the actual value of $J R C$ is slightly smaller than the designed value and the difference between them gets bigger when $J R C$ increases. Because the rougher the joint, the more sharp surface he has, and in the experiment because of the granularity of the cement mortar, the specimen cannot completely replicate the design surface form, resulting in the rougher the joint, the greater the error between the real value of JRC and the design value.

The JRC of joints can be calculated from a commonly adopted empirical equation [17]:

$$
\mathrm{JRC}=32.69+32.98 \log _{10} Z_{2},
$$

where the parameter $Z_{2}$ is given as 


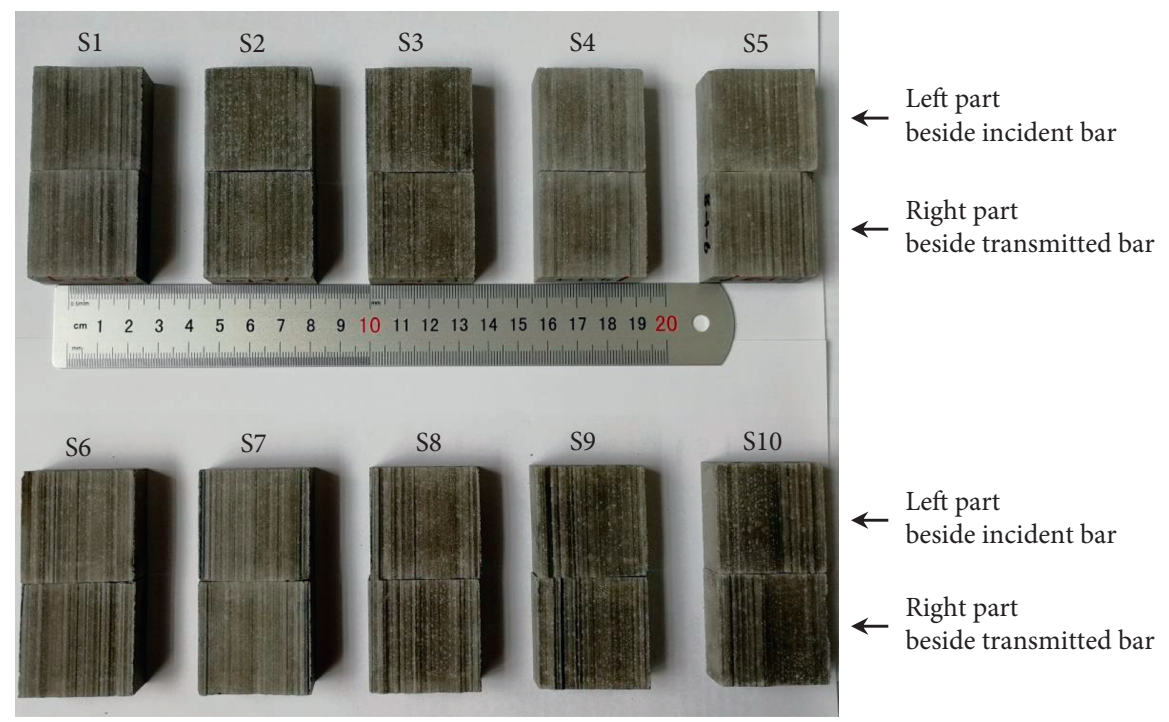

FIGURE 3: Jointed specimens with different JRC.

TABLE 1: The difference between the actual and designed values of $J R C$.

\begin{tabular}{|c|c|c|c|c|c|c|c|c|c|c|}
\hline Specimens & S1 & S2 & S3 & S4 & S5 & S6 & S7 & S8 & S9 & S10 \\
\hline f $J R$ & 0.703 & 2.904 & 5.216 & 7.106 & 9.235 & 11.02 & 12.83 & 15.80 & 16.96 & 19.18 \\
\hline Actual value of $J R C$ & 0.644 & 2.946 & 4.937 & 6.774 & 9.058 & 10.62 & 11.68 & 13.97 & 15.05 & 17.15 \\
\hline
\end{tabular}

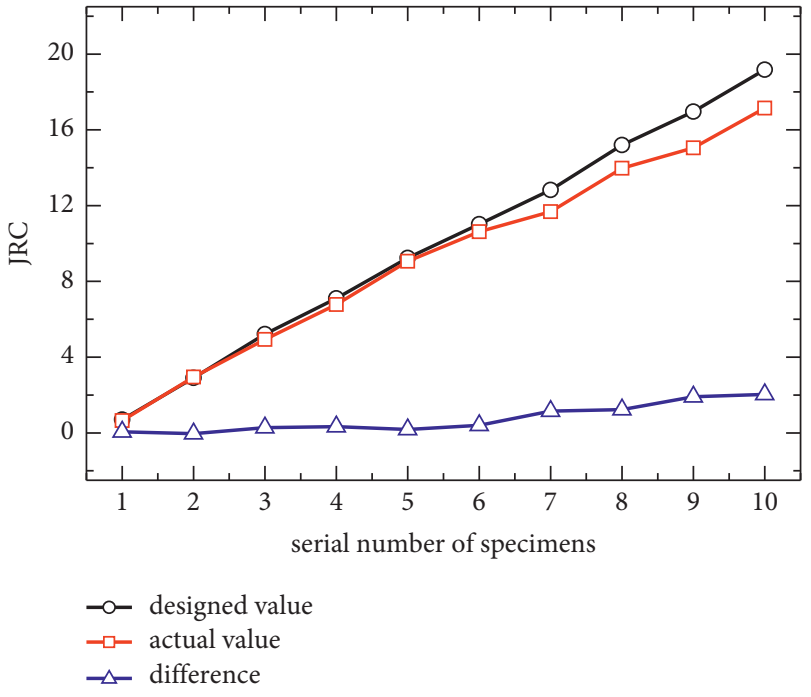

FIgURE 4: The difference between the actual and designed values of JRC.

$$
Z_{2}=\left[\frac{1}{m(\Delta x)^{2}} \sum_{i=1}^{m}\left(y_{i+1}-y_{i}\right)^{2}\right]^{\frac{1}{2}} \text {. }
$$

In this equation, $\triangle x$ denotes the small constant horizontal distance between two adjacent points along the profile lines, $m$ denotes the number of points on the profile lines and is related to the ratio between the horizontal distance of the profile line $D_{s}$ and horizontal distance interval $\triangle x$, that is, $m=1+\operatorname{integer}\left(D_{s} / \triangle x\right)$. The ' $y_{\mathrm{i}+1}-y_{\mathrm{i}}$ ' represents the amplitude difference between two adjacent points. As we can see, $Z_{2}$ is the root mean square of the first derivative of the profile. In this way, the $J R C$ of all specimens is ascertained.

2.3. Test Proceeding. A series of uniaxial compression tests on the specimens were carried out on SHPB apparatus. Before we placed each specimen coaxially between the incident and transmitted bars, a moderate amount of vaseline was smeared on the contact surfaces between the specimen and the bars to reduce the friction.

During the tests, launched by the dynamic loading device, the bullet impacted the incident bar, and consequently, the incident wave was generated and propagated along the incident bar. When the incident wave arrived at the specimen, reflected wave was caused and propagated back to the incident bar. Meanwhile, the specimen deformed. At this moment, the transmitted wave was generated on the interface and propagated along the transmitted bar. The waves, named as the incident, reflected, and transmitted waves, respectively, were measured by strain gauges glued on the incident and transmitted bars and then collected by the dynamic strain meter and stored by the oscilloscope.

\section{Data Processing}

A series of uniaxial compression tests on the specimens were carried out on the SHPB apparatus. The data collected from strain gauges provided the strains of the incident, reflected, and transmitted waves that are $\varepsilon_{i}, \varepsilon_{r}$, and $\varepsilon_{t}$, respectively. According to the one-dimensional wave propagation equation in the incident and transmitted bars, the stress $\sigma$, 
strain rate $\dot{\varepsilon}$, and strain $\varepsilon$ of the specimen can be calculated by the three waves, that is,

$$
\begin{aligned}
& \dot{\varepsilon}(t)=\frac{C_{0}}{l_{s}}\left(\varepsilon_{i}(t)-\varepsilon_{r}(t)-\varepsilon_{t}(t)\right), \\
& \varepsilon(t)=\frac{C_{0}}{l_{s}} \int_{0}^{t}\left(\varepsilon_{i}(t)-\varepsilon_{r}(t)-\varepsilon_{t}(t)\right) \mathrm{d} t, \\
& \sigma(t)=\frac{A_{0}}{2 A_{s}} E\left(\varepsilon_{i}(t)+\varepsilon_{r}(t)+\varepsilon_{t}(t)\right),
\end{aligned}
$$

where $l_{s}$ denotes the length of the specimen, $C_{0}$ denotes the longitudinal wave velocity in SHPB bars, and $A_{0}$, and $A_{s}$ are the cross-section areas of the bars and specimen, respectively.

Transmission and reflection coefficients are usually adopted to analyze the effect of joint or jointed rock mass on wave propagation. Here, the transmission coefficient is denoted as $T$, and the reflection coefficient is denoted as $R$, which is the ratio of the peak values of transmitted and reflected waves to that of the incident wave, respectively. Hence, $T$ and $R$ can be expressed as

$$
\begin{aligned}
& T=\frac{\max \left|\varepsilon_{t}\right|}{\max \left|\varepsilon_{i}\right|}, \\
& R=\frac{\max \left|\varepsilon_{r}\right|}{\max \left|\varepsilon_{i}\right|} .
\end{aligned}
$$

The theoretical deformation of a jointed specimen, $\Delta v$, includes the total deformation of rock blocks, $\Delta v_{r}$, and the closure of the joint, $\Delta v_{j}$, so we can get:

$$
\Delta v_{j}=\Delta v-\Delta v_{r}
$$

And the stiffness of the joint is the derivative of the stress-closure curves, which is

$$
k_{n}=\frac{\partial \sigma}{\partial \Delta v_{j}},
$$

where $\sigma$ is the stress on the specimen.

\section{Experimental Results and Analysis}

4.1. Test Parameters and Typical Strain Gauge Record. A series of experiments were performed on specimens with joints whose $J R C$ ranged from nearly 0 to almost 20. During the experiments, the incident, reflected, and transmitted waves were recorded by strain gauges glued on the incident and transmitted bars. All data were collected by the dynamic strain meter and stored by the oscilloscope. Figure 5 shows the typical stress waves of S0 and S7. The specimen S0 is an intact specimen without joint, and the specimen S7 contains a joint whose $J R C$ equals 11.68. The recorded signals of the other jointed specimens are similar to that of S7 and consequently not plotted here.

As we can see that when the incident wave crosses the specimen, part of them would be reflected to form the reflection wave and the others could transmit through it that contributes to transmission waves. Specifically, for the intact specimen S0, the maximum strain value of transmitted wave (1.02E-4) and the absolute maximum strain value of reflected wave $(9.2 \mathrm{E}-5)$ are 0.66 and 0.60 times of the maximum strain value of incident wave (1.53E-4), respectively. And for the jointed specimen $S 7$, the maximum strain value of transmitted wave (1.01E-4) and the absolute maximum strain value of reflected wave (1.86E-5) are 0.43 and 0.81 times of the maximum strain value of incident wave $(2.31 \mathrm{E}-4)$, respectively. Compared with the intact specimen S0, the jointed specimen S7 reflects more stress wave and less stress wave propagates through it.

4.2. Transmission and Reflection Coefficients. The transmission coefficient $T$ and the reflection coefficient $R$ can be calculated by equations (6) and (7). The relationships between transmission/reflection coefficients and $J R C$ are displayed in Figures 6 and 7. As we can see, the transmission coefficients generally increase with the rise of the $J R C$, and, on the contrary, the reflection coefficients generally decrease with the rise of the $J R C$. Two linear equations are adopted to fit the experiment data, which are $T=3.16 E-3 \times$ $J R C+4.05 E-1, R=-3.39 E-3 \times J R C+8.48 E-1$. Since the value of $J R C$ is between 0 and 20 , the transmission coefficient $T$ of the jointed specimens should vary from 0.41 to 0.47 , and the reflection coefficient $R$ of the jointed specimens should vary from 0.78 to 0.85 . As for the intact specimen S0, the transmission coefficient $T$ and the reflection coefficient $R$ are 0.66 and 0.60 , respectively, according to the waveform shown in Figure 5(a). Therefore, the transmission coefficient for wave propagation across an intact specimen is larger than any specimen with a joint, and correspondingly, the reflection coefficient of the intact specimen is smaller than that of all jointed specimens. Regardless of the roughness of the joint, the joint itself affects the stress wave propagation, and it makes more stress wave reflected and less stress wave transmitted through it. When the roughness of the joint is taken into consideration, it can be concluded from Figures 6 and 7 and the linear fitting equations that more stress wave would be transmitted and less stress wave would be reflected off the joint with rougher surfaces. Besides, considering the almost equal absolute values of the independent variable coefficient of the fitting equation for transmission and reflection coefficients, $J R C$ has the same extent of the effect on the reflection and transmission of stress waves propagating through the joints.

4.3. Stress-Strain Relations and Stiffness Analysis. According to data collected through the tests, the stress $\sigma$ and strain $\varepsilon$ of the specimen can be calculated by equations (3) to (5). Then we can get stress-strain relation curves of the intact specimen (S0) and jointed specimens (S1 to S10), which are shown in Figure 8. In the tests, the impact forces on all specimens were nearly the same, so the deformations of them were relatively comparable. It is evident from Figure 8 that the deformation of the jointed specimens is 


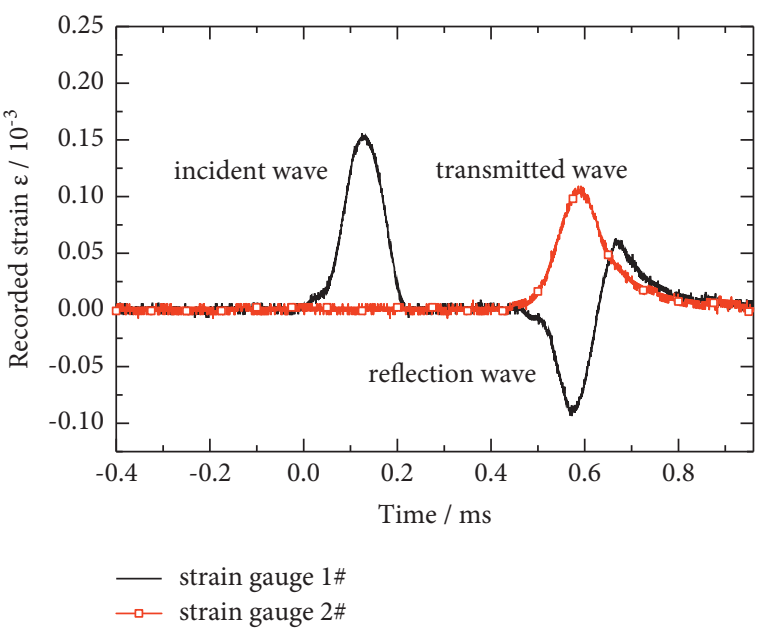

(a)

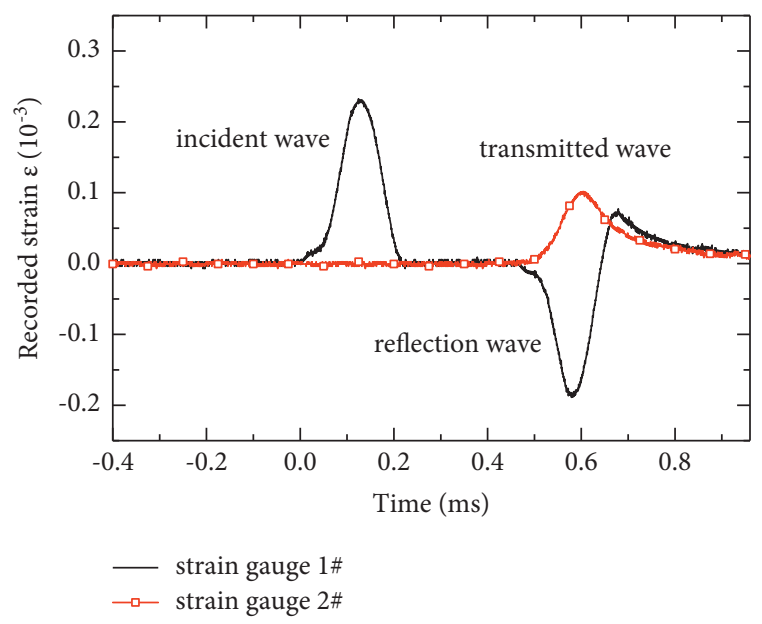

(b)

FIGURE 5: The recordings of strain gauges for specimen with and without joint. (a) The waveform of intact specimen S0. (b) The typical waveform of jointed specimen S7.

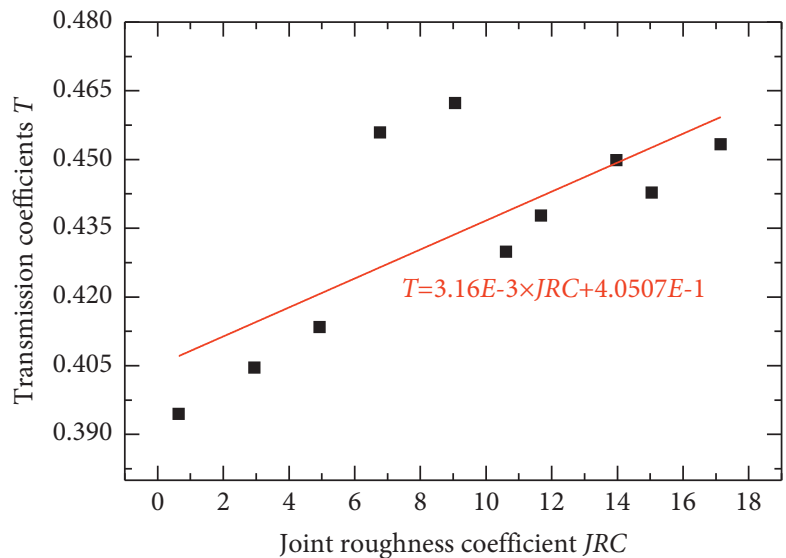

- transmission coefficients $T$

— linear fit

FIGURE 6: The variation of transmission coefficient with $J R C$ of specimen.

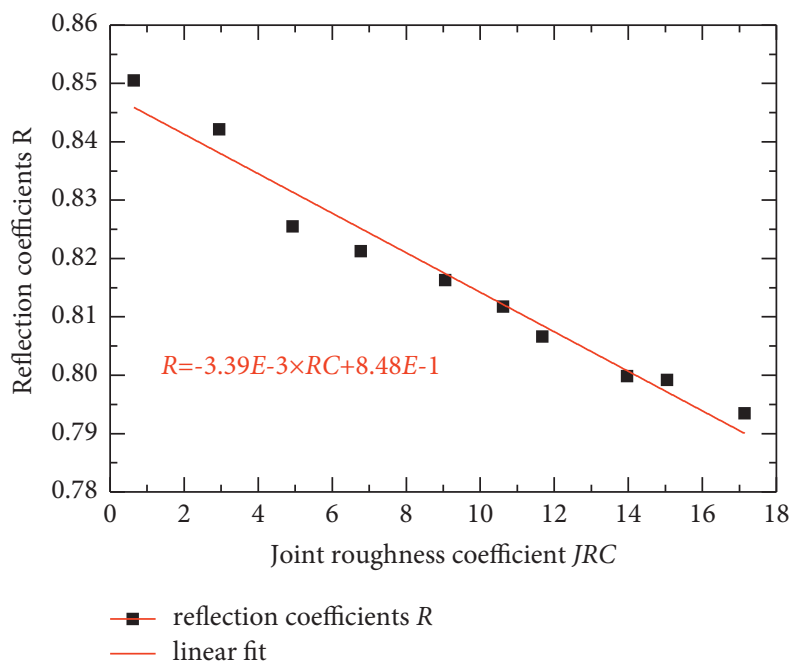

FIGURE 7: The variation of reflection coefficient with $J R C$ of specimen. 


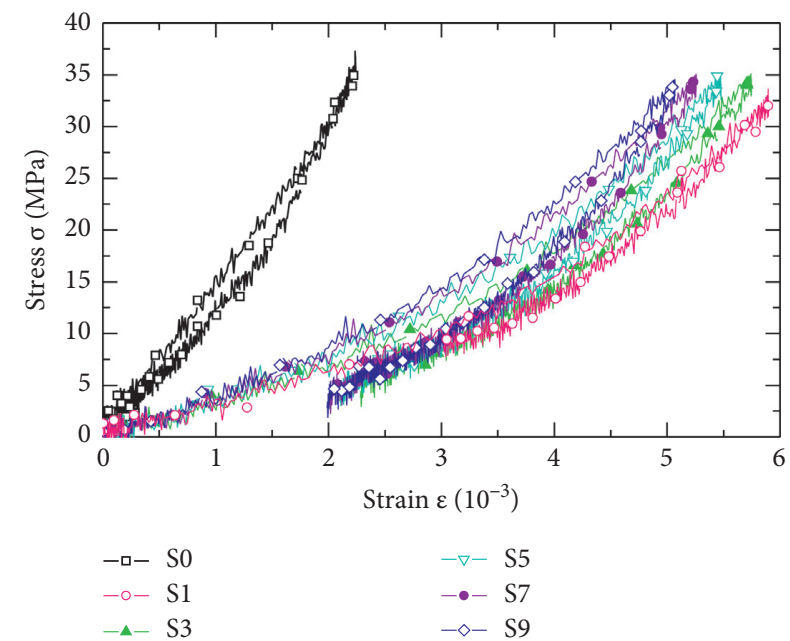

(a)

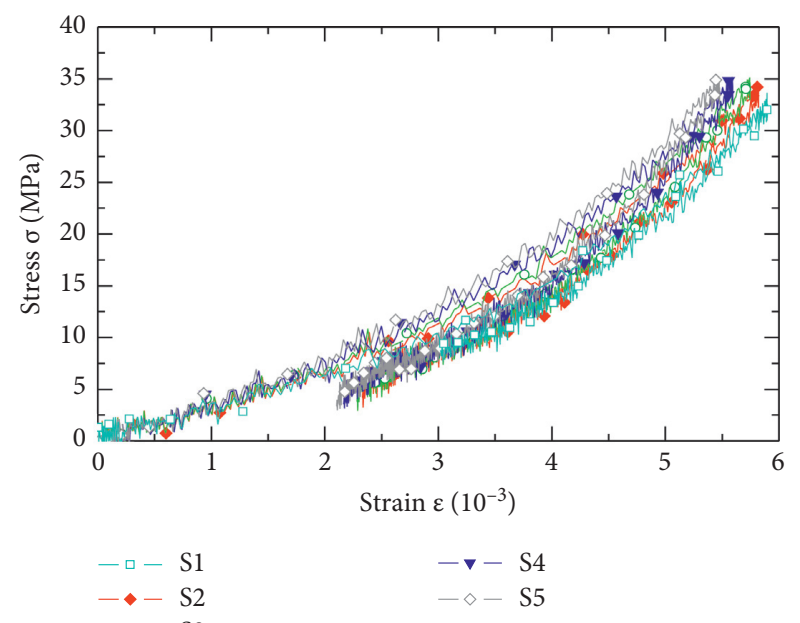

(c)

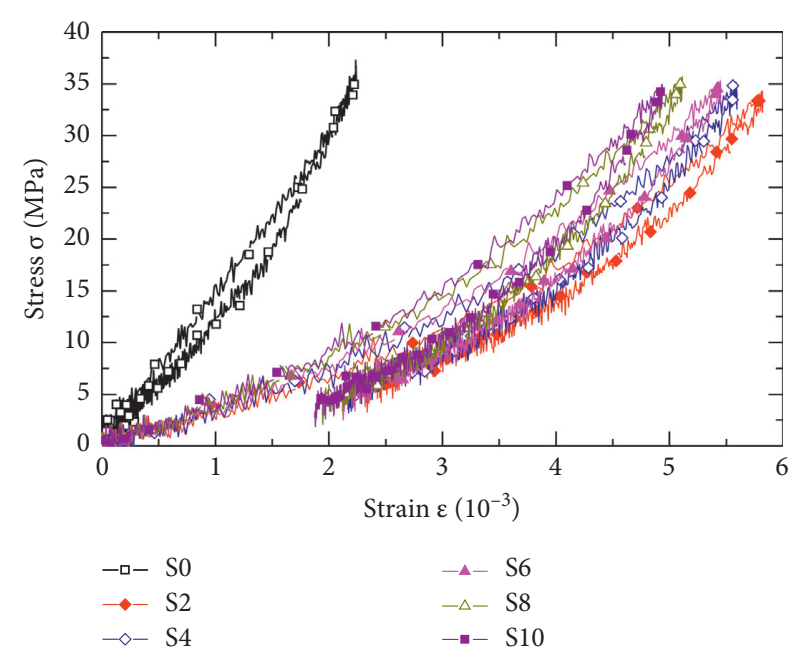

(b)

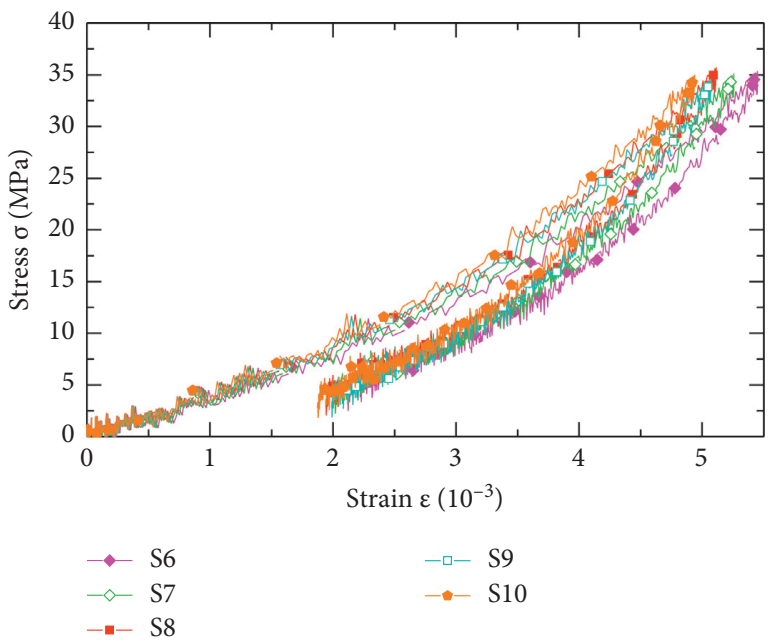

(d)

Figure 8: The stress-strain curves of specimens. (a) An intact specimen S0 and jointed specimens (S1, S3, S5, S7, S9); (b) an intact specimen S0 and jointed specimens (S2, S4, S6, S8, S10); (c) jointed specimens (S1, S2, S3, S4, S5); (d) jointed specimens (S6, S7, S8, S9, S10).

much larger than the intact one, to be exact, the minimum of the maximum strains of all jointed specimens $(4.94 \mathrm{E}-3)$ is more than twice the maximum strain of the intact one S0 (2.24E-3). The joint significantly enlarges the deformation of the specimen due to high compliance of the joint, which means the stiffness of the specimen would dramatically decreases because of the joints. When it comes to the compliance or the stiffness of the jointed specimens, it can be seen in Figure 8 that the slope of the stress-strain curves gradually increases as the joint gets rougher. To dig out the relationship between the stiffness and the roughness of the joint, we separated the deformation of the joint from the total deformation of the specimen. To this end, the stressstrain curves of the jointed specimens and intact specimen were first fitted with curves, and then after subtracting the deformation of the intact specimen from that of the jointed specimen by the fitted equations, the stress-closure curves of the joints with different roughness were obtained. Here, the intact specimen S0 and jointed specimen S2 are taken as the examples, which are shown in Figure 9. Considering the stress-closure curves were non-linear in the loading process, an exponential form as follows was taken to fit them using the least square regression method.

$$
\sigma=a[\exp (b \cdot \Delta v)-1]
$$

where $a$ and $b$ were parameters of the fitting equation. It can be seen from Figure 9 that the exponential form fits the curves of S0 and S2 well. By subtracting the normal deformation of the S0 from the deformation of the S2 under every same stress, we can get the stress-closure relation of the joint in S2 which is shown as the middle red curve in Figure 9. The stress-strain relation curves of other specimens are also fitted well with exponential equations, which are not shown as figures, and the parameters of the fitting curves are listed in Table 2.

The stress-closure relation curves of joints of all specimens are shown in Figure 10. In the figure, the curves resemble each other in shape, and slopes of all curves increase with the closure. However, the overall stiffness differs from 


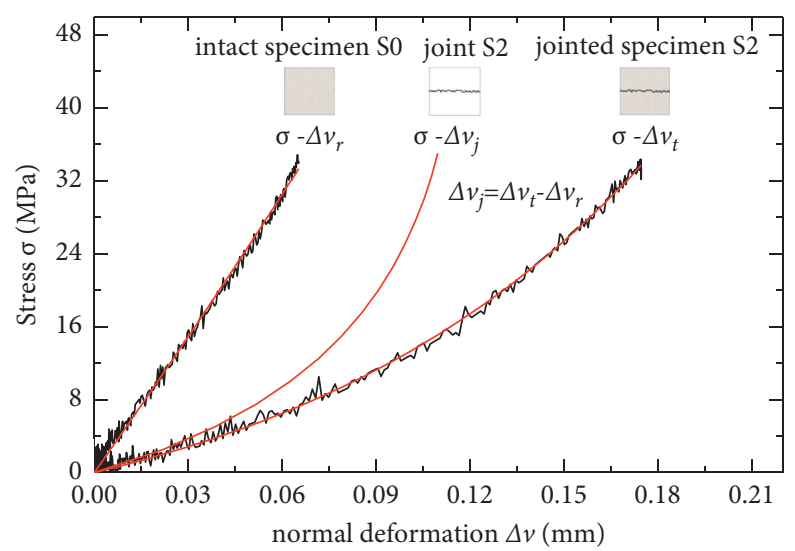

Figure 9: The deformation of specimen S2 under the normal stress.

TABLE 2: The parameters of stress-strain curve fitting equations.

\begin{tabular}{cccccccccccc}
\hline & S0 & S1 & S2 & S3 & S4 & S5 & S6 & S7 & S8 & S9 & S10 \\
\hline A & 331.44 & 8.66 & 9.54 & 9.93 & 10.97 & 11.67 & 11.44 & 11.53 & 12.23 & 12.85 & 13.13 \\
B & 1.47 & 8.76 & 8.65 & 8.66 & 8.44 & 8.34 & 8.53 & 8.77 & 8.81 & 8.50 & 8.67 \\
\hline
\end{tabular}

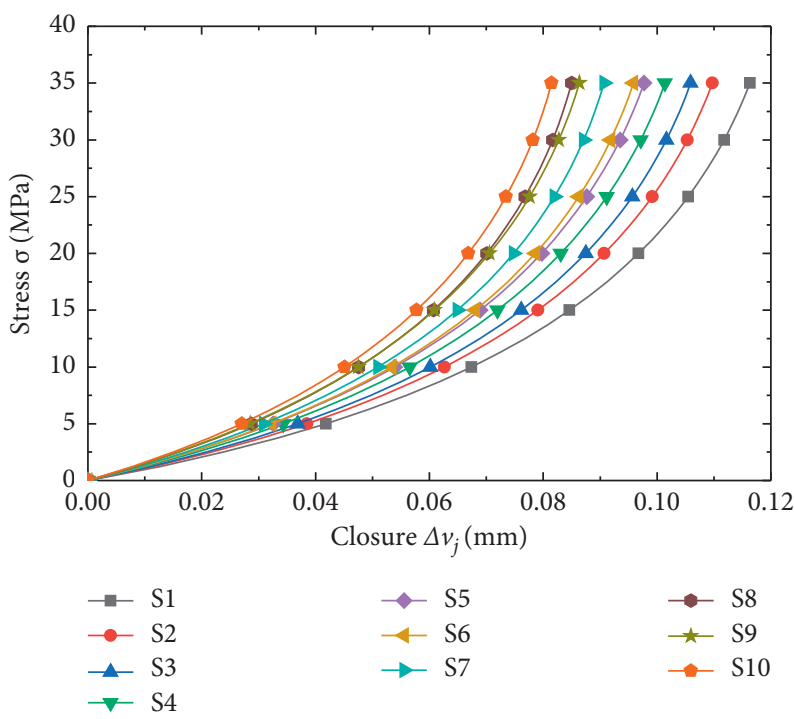

Figure 10: The stress-closure curves for joints of specimens S1-S10.

one to another, and there is a tendency that the stiffness of the joint increases when the joint wall has a high roughness. The initial stiffness of the joint $k_{\mathrm{ni}}$, as the reflection of overall stiffnesses, was calculated by the derivative of the curves at the origin point. Afterward, the relationship between the $k_{\mathrm{ni}}$ and $J R C$ was obtained and shown in Figure 10. There appears to be a positive relationship between the two variables. Hence, a linear equation was adopted to fit them, which is

$$
k_{\mathrm{ni}}=3.49 \times \mathrm{JRC}+88.30 .
$$

The normal stress-closure expression of the B-B model [6] is commonly adopted to cope with the issue of joint mechanical properties, which is

$$
\sigma=\frac{k_{\mathrm{ni}} \times \Delta v_{j}}{1-\Delta v_{j} / v_{\max }}
$$

where $\Delta v_{j}$ is the closure of the joint and the $v_{\max }$ is the maximum closure of the joint. The $k_{\text {ni }}$ of the jointed is a known coefficient that could be calculated by taking the derivative of the stress-closure curves at the beginning point. The equation.(12) is applied to fit the stress-closure curves of the joints to obtain the maximum closure $v_{\max }$. The fitting curves are shown in Figure 11, and the corresponding parameters of the equations of the fitting curves are displayed in Table.3. As we can see, the B-B model fits well with the stress-closure curves of joints, and the goodness of fit $\left(R^{2}\right)$ is 


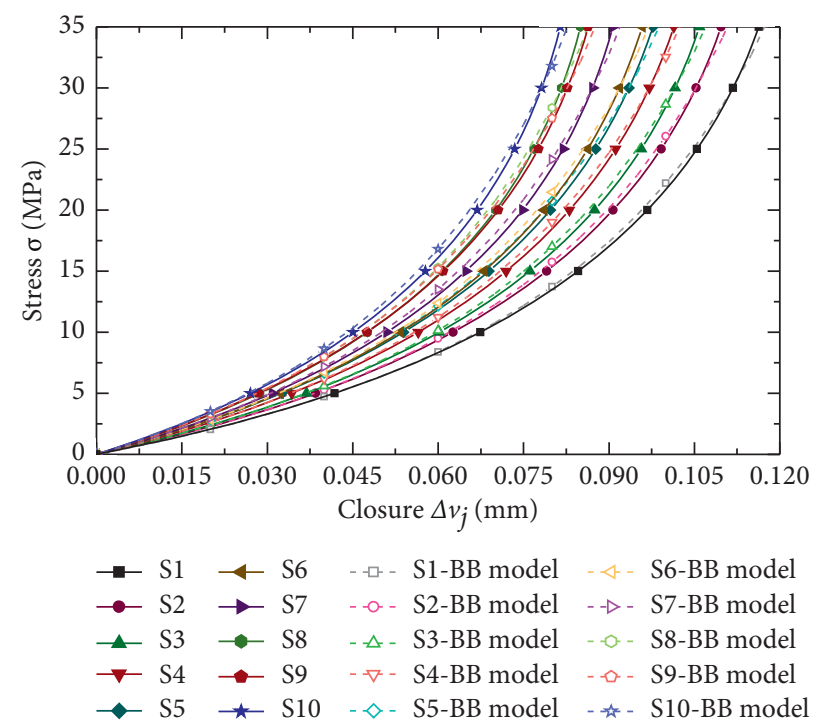

Figure 11: The fitting curves for stress-closure curves of joints S1-S10.

TABLE 3: The parameters of stress-closure curve fitting equations.

\begin{tabular}{lcccccccccc}
\hline & S1 & S2 & S3 & S4 & S5 & S6 & S7 & S8 & S9 & S10 \\
\hline$k_{n i}(\mathrm{GPa} / \mathrm{m})$ & 89.832 & 99.377 & 104.49 & 114.30 & 121.72 & 122.04 & 127.62 & 138.39 & 140.71 & 148.54 \\
$v_{\max }(\mathrm{mm})$ & 0.168 & 0.162 & 0.157 & 0.154 & 0.151 & 0.147 & 0.139 & 0.131 & 0.135 & 0.128 \\
$R^{2}$ & 0.9987 & 0.9985 & 0.9983 & 0.9982 & 0.9981 & 0.9978 & 0.997 & 0.9965 & 0.9972 & 0.9964 \\
\hline
\end{tabular}

all over 0.99 . Hence, the parameter $\left(v_{\max }\right)$ obtained from curve-fitting is credible to some extent and its variation with $J R C s$ of specimens is displayed in Figure 12. Obviously, the maximum closure of the joint $\left(v_{\max }\right)$ diminishes along with the increment of $J R C$. It, on the other hand, proves that the rougher joint has a higher stiffness in normal dynamic loading.

\section{Discussion}

5.1. Statement about the Dynamic Mechanical Behaviors of Specimens. The dynamic mechanical behaviors of specimens with and without joints are quite different. One of the differences lies in the deformation. As we can see in Figure 8, the jointed specimens ( $S 1$ to $\mathrm{S} 10$ ) deform greater than the specimen without joint (S0) under the same level of stress. Joints are commonly considered to be imperfectly bonded interface or slip interface, and the displacement across the joint is not continuous. The displacement difference across the joint is regarded as the deformation of the joint. Apart from the deformation of the rock-like block, extra deformation produced by the joint adds to the total deformation of the specimen. That is probably the main reason for the greatly increment in deformation of jointed specimen compared with the deformation of the intact specimen.

Another difference is the curvature of the stress-strain loading curve. The curvature of a curve is the rate of rotation of the tangential direction angle to the arc length for a point on the curve. Mathematically, it refers to the value of the degree of curve curvature. For the intact

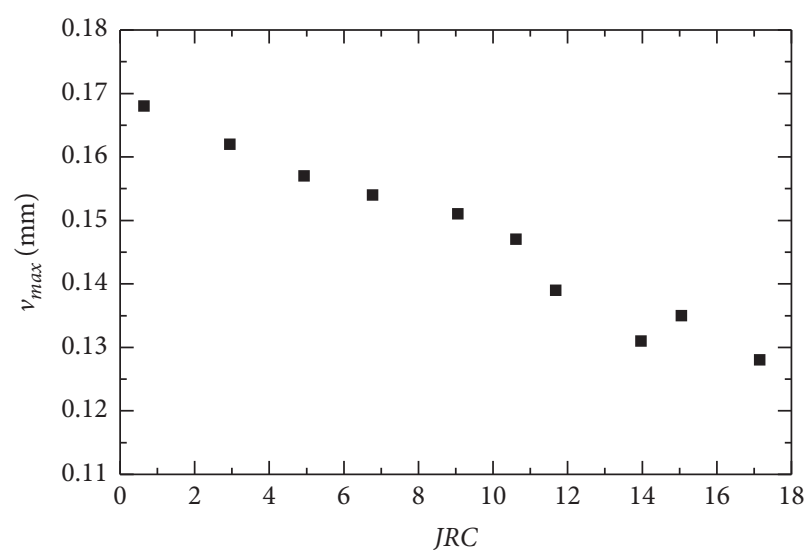

FIgURE 12: The variation of the joint maximum closure with JRC.

specimen, the relationship between stress and strain at the stage of loading is almost linear. For a jointed specimen, however, the stress-strain behavior is concave shaped with loading. In other words, the jointed specimens are nonlinear during the loading process. It is well understood that the intact rock block ( $\mathrm{S} 0)$ is in linear elastic deformation when the load is relatively small. Under a similar level of loading, the rock itself in the jointed specimen is in linearly elastic deformation. What induces the non-linear stressstrain relationship of the jointed specimen is the nonlinearity of joint in stress-strain relation. By subtracting the deformation of the intact specimen from that of the jointed specimen, the closure of joint is obtained, and then 
we can get its non-linear stress-closure curve shown in Figure 10. There has not been a comprehensive explanation about the non-linear nature of the joint stress-closure relationship. The reason could be that open microcracks of the joint start to close during the loading, making its modulus increase accordingly[21]. Another possible explanation is that the joint goes stiffening under normal stress because of an increase in the number of contacts between adjacent grains and asperities on opposite surfaces of joints [22].

5.2. Discussion on the Effect of the Joint Roughness. Besides, the roughness of the joint also influences the stressclosure relationship of joint. As it is shown in Figure 13, there is a positive linear relationship between the initial stiffness $k_{\mathrm{ni}}$ of the joint and the joint roughness JRC. On the opposite, another parameter of the joint B-B model, $v_{\max }$, has a negative relationship with JRC. That means that the rougher the rock joint is, the stiffer the joint is and more stress waves could pass through it. And correspondingly, the acoustic wave velocity of the specimen with rougher joint would be larger than that of the specimen with a flat joint. To identify this deduction, we performed the ultrasonic tests on mortar and granite specimens with flat and rough joints using the acoustic waves monitor (a type of RSM-SY6). And we found that the acoustic wave velocity of the mortar specimen with flat joint surface is about $2630 \mathrm{~m} / \mathrm{s}$, smaller than that of the mortar specimen with rough joint surface $(J R C=8.36)$ that is about $2800 \mathrm{~m} / \mathrm{s}$. Similarly, the acoustic wave velocity of the granite specimen with a flat joint surface is about $3410 \mathrm{~m} / \mathrm{s}$, smaller than that of the granite specimen with the rough joint surface $(J R C=8.36)$ that is about $3560 \mathrm{~m} / \mathrm{s}$. These two sets of jointed specimens were made in different ways. The mortar specimens were cast by printed mold. Granite specimens were carved by a stone milling machine (a type of LD $6060 \mathrm{CNC}$ ). No matter what way we choose to make those jointed specimens, the test results share similar results, which is the acoustic wave velocity of the specimen with a flat joint surface is smaller than that of the specimen with a rough joint surface. The outcome of ultrasonic tests supports the result of SHPB experiments on the jointed specimens. Also, the test outcome shares the same regularity with the results of the investigation done by $[6,23]$, which proves the validity of the result of the study.

How does the roughness of the joint affect the stress wave propagation? One possible factor could be the length of the joint profile line. As we know every surface is rough on some scale [14], two surfaces of the rock joint can never be $100 \%$ contacted even though the test specimens are all designed to be totally touched. But when the profile line of the joint becomes longer, the area of the joint surface is enlarged and the possible contact area would be also enlarged. So the rougher joint with larger contact area could let more stress wave pass by. With more contact area, the pressure on the joint surface would be lower. This is just a possible explanation. The intrinsic mechanism of influence of joint surface topography on the joint behavior under compression needs to be further investigated in the future.

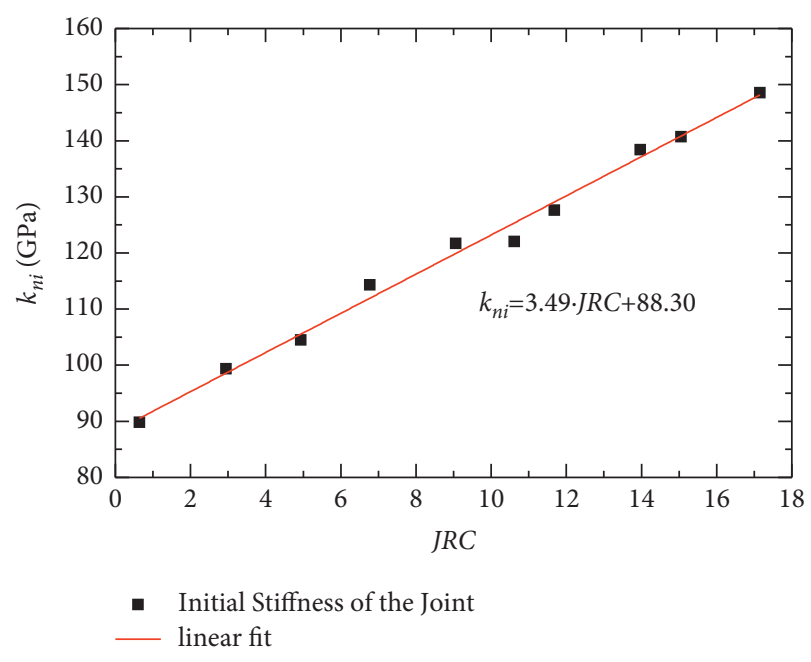

Figure 13: The relationship between the $k_{\mathrm{ni}}$ and $J R C$.

5.3. Reflection and Outlook on the Whole Experiment. Looking at the whole experiment, there are some disadvantages and limitations. As we know, the SHPB experiments technique is constructed on some basic assumptions, and one of them is that the stress field of the specimen is uniform along the length. But in actual tests, the joint surfaces of specimens are uneven and not perpendicular to the axis of the incident and transmitted bars. Consequently, even with plane compression wave propagating along the incident bars, reflected and transmitted waves upon the joints are not uniformly parallel to the axis, and a complex stress wave field is produced around the joint, which disobeys the assumption I mentioned above. As a result, the error occurs, and its effect on the outcome remains to be further investigated. The potential solution, such as numerical simulation or digital image correlation technique, may help in the analysis of the stress wave field around the joints. Apart from that, when the joint profile line is extremely flat/rugged, the value of $J R C$ calculated by the equation (1) would be negative/positive infinite, which exceed the range of the JRC (from 0 to 20). That is the limitation of this kind of definition of the JRC. So the situation when the joint that is way too flat or roughed is not included in this study. Though the concept of $J R C$ has been wildly used in rock engineering, it should be improved to suit more cases.

\section{Conclusions}

From the test results, the following conclusions can be drawn:

(1) When the stress wave propagates to the joint specimen, part of them is reflected, and the other is transmitted. The stress wave transmits more easily through the jointed specimens with higher JRC. Correspondingly, the reflection coefficient $R$ decreases with an increment of $J R C$. It can also be concluded that $J R C$ has the same extent of the effect on the reflection and transmission of stress waves. 
(2) As for the dynamic behavior of the joint, the stressclosure relation curves of joints fitted well with the $\mathrm{B}-\mathrm{B}$ model. The joint initial stiffness $\left(k_{\mathrm{ni}}\right)$ and the maximum closure $\left(v_{\max }\right)$ are two representative parameters. From the test results, we could find out that $k_{\mathrm{ni}}$ increases along with the increment of $J R C$. While $v_{\max }$ diminishes along with the increment of $J R C$.

\section{List of symbols}

$\begin{array}{ll}A_{0} \text { and } & \text { Cross-section areas of the SHPB bars and } \\ A_{s}: & \text { specimen, respectively } \\ C_{0}: & \text { Longitudinal wave velocity in SHPB bars } \\ D_{s}: & \text { Horizontal distance of the profile line } \\ E: & \text { Young's modulus of the SHPB bars } \\ H: & \text { Hurst exponent } \\ J R C: & \text { Joint roughness coefficient } \\ k_{n}: & \text { Joint normal stiffness } \\ k_{\mathrm{n}}: & \text { Joint normal initial stiffness } \\ l_{s}: & \text { Length of the specimen } \\ m: & \text { Number of points on the profile line } \\ R: & \text { Reflection coefficient } \\ S F: & \text { Structure function } \\ t: & \text { Time } \\ T: & \text { Transmission coefficient } \\ \Delta v: & \text { Deformation of the specimen } \\ \Delta v_{r}: & \text { Deformation of rock blocks } \\ \Delta v_{j}: & \text { Closure of the joint } \\ v_{\text {max }}: & \text { The maximum value of the joint closure } \\ y_{i}: & \text { Vertical height of the point on the profile line of } \\ Z_{2}: & \text { joint surface } \\ & \text { Root mean square of the first derivative of the } \\ \rho: & \text { profile line } \\ \sigma: & \text { Density of the SHPB bars } \\ \varepsilon: & \text { Stress on the specimen } \\ \dot{\varepsilon}: & \text { Strain of the specimen } \\ \varepsilon_{i}, \varepsilon_{r} \text { and } & \text { Strain rate of the specimen } \\ \varepsilon_{t}: & \text { Strains caused by incident, reflected, and } \\ & \text { transmitted waves, respectively. }\end{array}$

\section{Data Availability}

The data used to support the findings of this study are included within the article.

\section{Conflicts of Interest}

The authors declare no conflicts of interest.

\section{Acknowledgments}

This work was supported by the Chinese National Science Research Fund (grant nos. 41525009, 41831281, and 51439008) and National Science Foundation of Hebei Province of China (grant no. E2019210291).

\section{References}

[1] L. Haibo, X. Xiang, L. Jianchun, Z. Jian, L. Bo, and L. Yaqun, "Rock damage control in bedrock blasting excavation for a nuclear power plant," International Journal of Rock Mechanics and Mining Sciences, vol. 48, no. 2, pp. 210-218, 2011.

[2] L. L. Gu, Z. Wang, Q. Huang, G. L. Ye, and F. Zhang, "Numerical investigation into ground treatment to mitigate the permanent train-induced deformation of pile-raft-soft soil system," Transportation Geotechnics, vol. 24, Article ID 100368, 2020.

[3] N. Barton, "The shear strength of rock and rock joints," International Journal of Rock Mechanics and Mining Science \& Geomechanics Abstracts, vol. 13, no. 9, pp. 255-279, 1976.

[4] N. G. W. Cook, "Natural joints in rock: mechanical, hydraulic and seismic behaviour and properties under normal stress," International Journal of Rock Mechanics and Mining Science \& Geomechanics Abstracts, vol. 29, no. 3, pp. 198-223, 1992.

[5] W. Zhao, R. Huang, and M. Yan, "Study on the deformation and failure modes of rock mass containing concentrated parallel joints with different spacing and number based on smooth joint model in pfc," Arabian Journal of Geosciences, vol. 8, no. 10, pp. 7887-7897, 2015.

[6] S. C. Bandis, A. C. Lumsden, and N. R. Barton, "Fundamentals of rock joint deformation," International Journal of Rock Mechanics and Mining Science of Geomechanics Abstracts, vol. 20, no. 6, pp. 249-268, 1983.

[7] L. J. Pyrak-Nolte, L. R. Myer, and N. G. W. Cook, "Transmission of seismic waves across single natural fractures," Journal of Geophysical Research, vol. 95, no. B6, pp. 8617$8638,1990$.

[8] J. Zhao, J. G. Cai, X. B. Zhao, and H. B. Li, “Dynamic model of fracture normal behaviour and application to prediction of stress wave attenuation across fractures," Rock Mechanics and Rock Engineering, vol. 41, no. 5, pp. 671-693, 2008.

[9] M. Schoenberg, "Elastic wave behavior across linear slip interfaces," Journal of the Acoustical Society of America, vol. 68, no. 5, pp. 1516-1521, 1980.

[10] J. Li, G. Ma, and J. Zhao, "An equivalent viscoelastic model for rock mass with parallel joints," Journal of Geophysical Research Solid Earth, vol. 115, no. B3, pp. 1923-1941, 2010.

[11] Z. Wang, L. Gu, Q. Zhang, S. Yue, and G. Zhang, "Creep characteristics and prediction of creep failure of rock discontinuities under shearing conditions," International Journal of Earth Sciences, vol. 109, no. 3, pp. 945-958, 2020.

[12] L. Myer, D. Hopkins, and N. Cook, "Effects of contact area of an interface on acoustic wave transmission characteristics," in Proceeding of the 26th US Symposium on Rock Mechanics (USRMS), American Rock Mechanics Association, Rapid City, South Dakota, June 1985.

[13] D. L. Hopkins, The Effect of Surface Roughness on Joint Stiffness, Aperture, and Acoustic Wave Propagation, Ph. D University of California, Berkeley, 1991.

[14] D. L. Hopkins, "The implications of joint deformation in analyzing the properties and behavior of fractured rock masses, underground excavations, and faults," International Journal of Rock Mechanics and Mining Sciences, vol. 37, no. 1, pp. 175-202, 2000.

[15] N. Barton and V. Choubey, "The shear strength of rock joints in theory and practice," Rock Mechanics and Rock Engineering, vol. 10, no. 1, pp. 1-54, 1977.

[16] R. Tse and D. M. Cruden, "Estimating joint roughness coefficients," International Journal of Rock Mechanics and 
Mining Science \& Geomechanics Abstracts, vol. 16, no. 5, pp. 303-307, 1979.

[17] Z. Y. Yang, S. C. Lo, and C. C. Di, "Reassessing the joint roughness coefficient (jrc) estimation using z 2," Rock Mechanics and Rock Engineering, vol. 34, no. 3, pp. 243-251, 2001.

[18] A. Misra and O. Marangos, "Rock-joint micromechanics: relationship of roughness to closure and wave propagation," International Journal of Geomechanics, vol. 11, no. 6, pp. 431-439, 2010.

[19] J. C. Li, L. F. Rong, H. B. Li, and S. N. Hong, "An shpb test study on stress wave energy attenuation in jointed rock masses," Rock Mechanics and Rock Engineering, vol. 52, no. 4, 2018.

[20] H. F. Xu, Y. R. Li, X. Y. Liu, and T. P. Liao, "Fractal simulation of joint profiles and relationship between jrc and fractal dimension," Chinese Journal of Rock Mechanics and Engineering, vol. 21, no. 11, pp. 1663-1666, 2002.

[21] J. B. Walsh and M. A. Grosenbaugh, "New model for analyzing the effect of fractures on compressibility," Journal of Geophysical Research, vol. 84, no. NB7, pp. 3532-3536, 1979.

[22] S. Glubokovskikh, B. Gurevich, M. Lebedev, V. Mikhaltsevitch, and S. Tan, "Effect of asperities on stress dependency of elastic properties of cracked rocks," International Journal of Engineering Science, vol. 98, pp. 116-125, 2016.

[23] L. Yexue, X. Heping, Z. Zheming, F. Huadong, and Y. Jianjun, "Study on rules of transmission and reflection of stress wave across fractal joint," Chinese Journal of Rock Mechanics and Engineering, vol. 28, no. 1, pp. 120-129, 2009. 